\title{
Pregabalin for decreasing pancreatic pain in chronic pancreatitis (Review)
}

\author{
Gurusamy KS, Lusuku C, Davidson BR
}

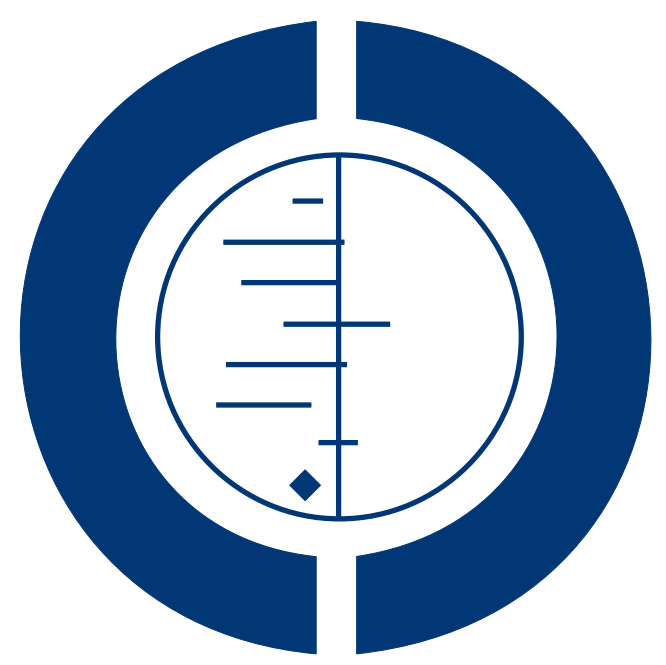

\section{THE COCHRANE COLLABORATION $^{\circledR}$}

This is a reprint of a Cochrane review, prepared and maintained by The Cochrane Collaboration and published in The Cochrane Library 2016, Issue 2

http://www.thecochranelibrary.com

\section{WILEY}

Pregabalin for decreasing pancreatic pain in chronic pancreatitis (Review)

Copyright @ 2016 The Cochrane Collaboration. Published by John Wiley \& Sons, Ltd. 
TABLE OF CONTENTS

HEADER . . . . . . . . . . . . . . . . . . . . . . . . . . . . . . . . . . . . . . . 1

ABSTRACT . . . . . . . . . . . . . . . . . . . . . . . . . . . . . . . . . . . . . . . . . . . . . . . . . . 1

PLAIN LANGUAGE SUMMARY . . . . . . . . . . . . . . . . . . . . . . . . . . . . . . . . . . . .

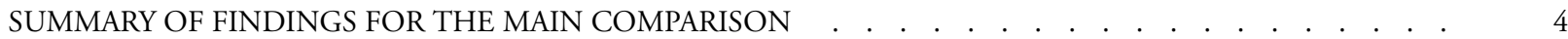

BACKGROUND . . . . . . . . . . . . . . . . . . . . . . . . . . . . . . . . . . . . . 6

OBJECTIVES . . . . . . . . . . . . . . . . . . . . . . . . . . . . . . . . . . . . . . . . . . . . .

METHODS . . . . . . . . . . . . . . . . . . . . . . . . . . . . . . . . . . . . . .

RESUlTS . . . . . . . . . . . . . . . . . . . . . . . . . . . . . . . . . . . . 10

Figure 1. . . . . . . . . . . . . . . . . . . . . . . . . . . . . . . . . . . . . . 12

Figure 2. . . . . . . . . . . . . . . . . . . . . . . . . . . . . . . . . . . . . . . . . . .

DISCUSSION . . . . . . . . . . . . . . . . . . . . . . . . . . . . . . . . . . . . . 15

AUTHORS' CONCLUSIONS . . . . . . . . . . . . . . . . . . . . . . . . . . . . . . . . . . . . . . .

ACKNOWLEDGEMENTS . . . . . . . . . . . . . . . . . . . . . . . . . . . . . . . . . . . $\quad .17$

REFERENCES . . . . . . . . . . . . . . . . . . . . . . . . . . . . . . . . . . . . . . 17

CHARACTERISTICS OF STUDIES . . . . . . . . . . . . . . . . . . . . . . . . . . . . . . . . . . . . .

DATA AND ANALYSES . . . . . . . . . . . . . . . . . . . . . . . . . . . . . . . . . . . 22

Analysis 1.1. Comparison 1 Pregabalin versus placebo, Outcome 1 Health-related quality of life (short-term). . . . . 22

Analysis 1.2. Comparison 1 Pregabalin versus placebo, Outcome 2 Serious adverse events. . . . . . . . . . . . . $\quad 23$

Analysis 1.3. Comparison 1 Pregabalin versus placebo, Outcome 3 Opiate analgesic requirement in mg (short-term). 23

Analysis 1.4. Comparison 1 Pregabalin versus placebo, Outcome 4 Adverse events. . . . . . . . . . . . . . . . 24

Analysis 1.5. Comparison 1 Pregabalin versus placebo, Outcome 5 Pain scores (short-term). . . . . . . . . . . 24

Analysis 1.6. Comparison 1 Pregabalin versus placebo, Outcome 6 Hospital admissions. . . . . . . . . . . . . 25

APPENDICES . . . . . . . . . . . . . . . . . . . . . . . . . . . . . . . . . . . . . 25

CONTRIBUTIONS OF AUTHORS . . . . . . . . . . . . . . . . . . . . . . . . . . . . . . . . . . . 29

DECLARATIONS OF INTEREST . . . . . . . . . . . . . . . . . . . . . . . . . . . . . . . . 29



DIFFERENCES BETWEEN PROTOCOL AND REVIEW . . . . . . . . . . . . . . . . . . . . . . . 30

Pregabalin for decreasing pancreatic pain in chronic pancreatitis (Review)

Copyright @ 2016 The Cochrane Collaboration. Published by John Wiley \& Sons, Ltd. 


\title{
[Intervention Review] \\ Pregabalin for decreasing pancreatic pain in chronic pancreatitis
}

\author{
Kurinchi Selvan Gurusamy ${ }^{1}$, Charnelle Lusuku² ${ }^{2}$, Brian R Davidson ${ }^{1}$ \\ ${ }^{1}$ Department of Surgery, Royal Free Campus, UCL Medical School, London, UK. ${ }^{2}$ School of Medicine, The University of Nottingham, \\ Nottingham, UK \\ Contact address: Kurinchi Selvan Gurusamy, Department of Surgery, Royal Free Campus, UCL Medical School, Royal Free Hospital, \\ Rowland Hill Street, London, NW3 2PF, UK. k.gurusamy@ucl.ac.uk.
}

Editorial group: Cochrane Upper GI and Pancreatic Diseases Group.

Publication status and date: New, published in Issue 2, 2016.

Review content assessed as up-to-date: 22 June 2015.

Citation: Gurusamy KS, Lusuku C, Davidson BR. Pregabalin for decreasing pancreatic pain in chronic pancreatitis. Cochrane Database of Systematic Reviews 2016, Issue 2. Art. No.: CD011522. DOI: 10.1002/14651858.CD011522.pub2.

Copyright (C) 2016 The Cochrane Collaboration. Published by John Wiley \& Sons, Ltd.

\begin{abstract}
A B S T R A C T
Background

Chronic abdominal pain is one of the major symptoms in people with chronic pancreatitis. The role of pregabalin in people with chronic pancreatic pain due to chronic pancreatitis is uncertain.
\end{abstract}

\section{Objectives}

To assess the benefits and harms of pregabalin in people with chronic abdominal pain due to chronic pancreatitis.

\section{Search methods}

We searched the Cochrane Central Register of Controlled Trials (CENTRAL) in The Cochrane Library 2015, issue 6, and MEDLINE, EMBASE, Science Citation Index Expanded, trials registers until June 2015. We also searched the references of included trials to identify further trials.

\section{Selection criteria}

We considered only randomised controlled trials (RCT) performed in people with chronic pancreatic pain due to chronic pancreatitis, irrespective of language, blinding, or publication status for inclusion in the review.

\section{Data collection and analysis}

Two review authors independently identified trials and independently extracted data. We calculated the risk ratio (RR) or mean difference (MD) with 95\% confidence intervals (CI) with RevMan 5, based on intention-to-treat analysis.

\section{Main results}

Only one study, funded by Pfizer, met the inclusion criteria for the review. A total of 64 participants (with chronic pain due to chronic pancreatitis) were randomly assigned to receive escalating doses of pregabalin (150 mg per day to $600 \mathrm{mg}$ per day; 34 participants) or matching placebo (30 participants). Participants received pregabalin or placebo for three weeks on an outpatient basis; the outcomes were measured at the end of the treatment (i.e. three weeks from commencement of treatment). Potential participants taking concomitant analgesic medication and expected to stay on a stable regime during the trial were allowed to enter the study. This trial was at low risk of bias. The overall quality of evidence was low or moderate.

Pregabalin for decreasing pancreatic pain in chronic pancreatitis (Review)

Copyright (๑) 2016 The Cochrane Collaboration. Published by John Wiley \& Sons, Ltd. 
Only the short-term outcomes were available in this trial. The medium and long-term outcomes, number of work days lost, and length of hospital stay due to admissions for pain control were not available. This trial found that the changes in opiate use (MD -26.00 mg; 95\% CI -47.36 to -4.64 ; participants $=64$; moderate-quality evidence), and pain score percentage changes from baseline (MD -12.00 ; $95 \%$ CI -21.82 to -2.18 ; participants $=64$; moderate-quality evidence) were better in participants taking pregabalin compared to those taking placebo. This trial also found that there were more adverse events in participants taking pregabalin compared to those taking placebo (RR 1.71; 95\% CI 1.20 to 2.43; participants =64). The differences between pregabalin and placebo were imprecise for shortterm health-related quality of life measured with the EORTC CLQ-30 questionnaire (MD 11.40; 95\% CI -3.28 to 26.08; participants = 64; moderate-quality evidence), proportion of people with serious adverse events (RR 1.76; 95\% CI 0.35 to 8.96; participants = 64; low-quality evidence), and proportion of people requiring hospital admissions (RR 0.44; 95\% CI 0.04 to 4.62; participants = 64; low quality evidence).

\section{Authors' conclusions}

Based on low- to moderate-quality evidence, short-term use of pregabalin decreases short-term opiate use, and short-term pain scores, but increases the adverse events compared to placebo, in people with chronic pain due to chronic pancreatitis. The clinical implication of the decreases in short-term opiate use and short-term pain scores is not known.

Future trials assessing the role of pregabalin in decreasing chronic pain in chronic pancreatitis should assess the medium- or long-term effects of pregabalin and should include outcomes such as, quality of life, treatment-related adverse events, number of work days lost, number of hospital admissions, and the length of hospital stay, in addition to pain scores, to assess the clinical and socioeconomic impact.

\section{PLAIN LANGUAGE SUMMARY}

Pregabalin for decreasing abdominal pain in people with chronic pancreatitis

\section{Review question}

Is pregabalin useful in decreasing abdominal pain in people with chronic pancreatitis?

\section{Background}

The pancreas is an abdominal organ that secretes several digestive enzymes into the pancreatic ductal system, which empties into the small bowel. It also comprises the Islets of Langerhans, which secrete several hormones, including insulin. Chronic pancreatitis is longstanding and progressive inflammation of the pancreas resulting in destruction and replacement of pancreatic tissue with fibrous tissue. This may lead to a shortage of digestive enzymes and insulin (helps regulate blood sugar), leading to diabetes (a lifelong condition in which a person's blood sugar level becomes too high). Alcohol is considered the main cause but others include: smoking, some drugs, and a variety of other disorders. Chronic abdominal pain is the major symptom of chronic pancreatitis. The pain is usually in the upper abdomen and is usually described as deep, penetrating, and radiating to the back. Various theories exist about the reason for pain in chronic pancreatitis. One theory is that the disease process affects the nerves supplying the pancreas. Pregabalin inhibits the transmission of pain through the nerves. Pregabalin may decrease pain in people with chronic pancreatitis, but may also produce a number of side-effects. Some common side-effects include: excessive sleepiness, blurred vision, double vision, dry mouth, constipation, vomiting, excessive wind, feeling excited, confusion, reduced sexual desire, irritability, feeling dizzy, feeling unsteady, tremors, speech difficulty, tingling or pricking ('pins and needles') sensation, and disturbances of attention and memory. Less frequent, but serious adverse events include: fainting episodes, heart failure, and reversible kidney failure. This review included all studies 22 June 2015 , on the benefits and harms of using pregabalin to treat chronic pain in people with chronic pancreatitis.

\section{Study characteristics}

We only found one study funded by Pfizer that met our inclusion criteria. A total of 64 participants with chronic pain due to chronic pancreatitis received either increasing doses of pregabalin (150 mg per day to $600 \mathrm{mg}$ per day; 34 participants) or matching placebo (sham treatment; 30 participants) on an outpatient basis. The decision about whether a participant received pregabalin or placebo was made using methods similar to toss of a coin, to ensure that the participants in the two groups were similar. Participants received pregabalin or placebo for three weeks, then the outcomes were measured. Potential participants taking other pain-killers were allowed to take part in the study. Thus, this trial evaluates the role of pregabalin in addition to other analgesics for decreasing chronic abdominal pain due to chronic pancreatitis.

Pregabalin for decreasing pancreatic pain in chronic pancreatitis (Review)

Copyright () 2016 The Cochrane Collaboration. Published by John Wiley \& Sons, Ltd. 


\section{Key results}

Only the short-term outcomes were available in this trial. This trial found that the changes in opiate use (drugs similar to morphine), and pain scores from baseline were better in participants taking pregabalin compared to those taking placebo. It was not clear whether these changes had a significant impact on the life of the participants. This trial also found that there were more side-effects in participants taking pregabalin compared to those taking placebo. The differences between pregabalin and placebo were imprecise for short-term health-related quality of life, percentage of people with serious side-effects, and percentage of people requiring hospital admissions.

Medium- and long-term outcomes, number of work days lost, and length of hospital stay were not available in this trial.

\section{Quality of the evidence}

The quality of evidence was low or moderate. As a result, further studies are required on this topic. 


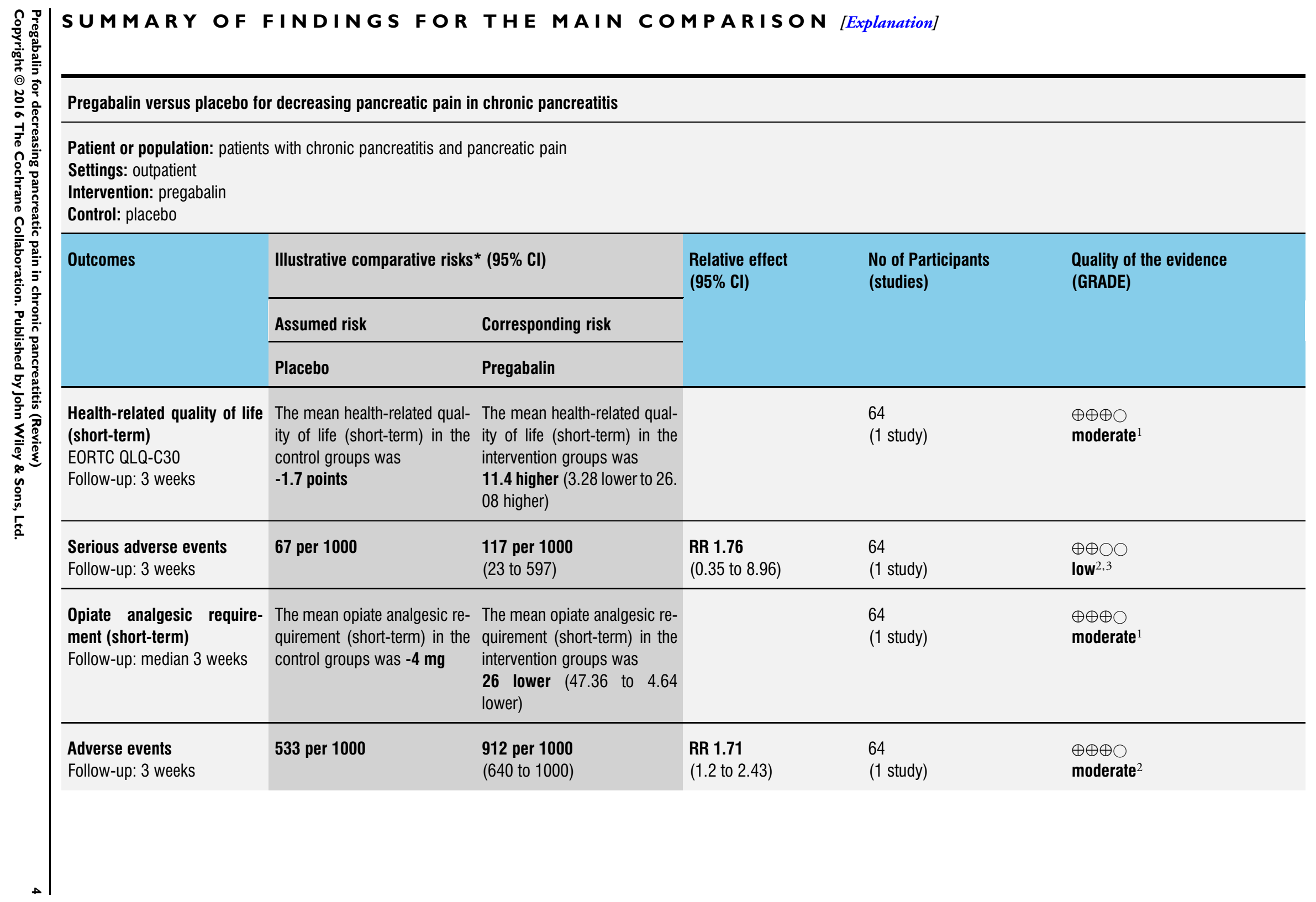




\begin{tabular}{|c|c|c|c|c|c|}
\hline $\begin{array}{l}\text { Pain scores (short-term) } \\
\text { Follow-up: } 3 \text { weeks }\end{array}$ & $\begin{array}{l}\text { The mean pain scores (short- } \\
\text { term) in the control groups } \\
\text { was }-24 \text { percentage change } \\
\text { from baseline }\end{array}$ & $\begin{array}{l}\text { The mean pain scores (short- } \\
\text { term) in the intervention } \\
\text { groups was } \\
12 \text { lower ( } 22 \text { to } 2 \text { lower) }\end{array}$ & & $\begin{array}{l}64 \\
\text { (1 study) }\end{array}$ & $\begin{array}{l}\oplus \oplus \oplus \bigcirc \\
\text { moderate }{ }^{1}\end{array}$ \\
\hline $\begin{array}{l}\text { Hospital admissions } \\
\text { Follow-up: } 3 \text { weeks }\end{array}$ & 67 per 1000 & $\begin{array}{l}29 \text { per } 1000 \\
(3 \text { to } 308)\end{array}$ & $\begin{array}{l}\text { RR } \mathbf{0 . 4 4} \\
\text { (0.04 to } 4.62)\end{array}$ & $\begin{array}{l}64 \\
(1 \text { study) }\end{array}$ & $\begin{array}{l}\oplus \oplus \bigcirc \bigcirc \\
\text { low }^{2,3}\end{array}$ \\
\hline
\end{tabular}

The only trial included in this review did not report medium or long-term outcomes and did not report the number of work days lost and length of hospital stay (0lesen 2011).

Although the risk of bias in the trial was low and the quality of evidence is low to moderate for short-term outcomes, the short duration of the trial and follow-up (i.e. medium to long-term outcomes were not available) and the lack of important clinical outcomes such as work days lost and length of hospital stay cause a major threat to external validity of the results as chronic pancreatitis is a long-standing disorder and the treatment is aimed only at symptomatic control of chronic pancreatitis. As a result, the effectiveness and treatment-related complications may be different between the short-term and the medium-term or long-term

*The basis for the assumed risk is the control group risk in the only study. The corresponding risk (and its $95 \%$ confidence interval) is based on the assumed risk in the comparison group and the relative effect of the intervention (and its $95 \% \mathrm{Cl}$ ).

Cl: Confidence interval; RR: Risk ratio.

GRADE Working Group grades of evidence

High quality: Further research is very unlikely to change our confidence in the estimate of effect.

Moderate quality: Further research is likely to have an important impact on our confidence in the estimate of effect and may change the estimate.

Low quality: Further research is very likely to have an important impact on our confidence in the estimate of effect and is likely to change the estimate.

Very low quality: We are very uncertain about the estimate.

${ }^{1}$ There were fewer than 400 participants included in both groups (imprecision).

2 There were fewer than 300 events in both groups (imprecision).

${ }^{3}$ The confidence intervals 1 and 0.75 or 1.25 or both (imprecision). 


\section{B A C K G R O U N D}

\section{Description of the condition}

Please see the glossary of terms in Appendix 1.

The pancreas is an abdominal organ that secretes several digestive enzymes into the pancreatic ductal system, which empties into the small bowel. It also comprises the Islets of Langerhans, which secrete several hormones, including insulin (NCBI 2014). Chronic pancreatitis is long-standing and progressive inflammation of the pancreas resulting in destruction and replacement of pancreatic tissue with fibrous tissue (structural deformity) (Braganza 2011). This may lead to the functional deformity of exocrine pancreatic insufficiency and endocrine pancreatic insufficiency (diabetes) ( Braganza 2011). Although previously considered as distinct from acute pancreatitis (since the pancreas returns to normal after an attack of acute pancreatitis), chronic pancreatitis is now considered to belong to the spectrum of pancreatitis disorders that include acute pancreatitis and acute recurrent pancreatitis, because of the overlapping aetiology and symptoms (Braganza 2011).

The annual incidence of chronic pancreatitis ranges from 1.5 to 7.9 per 100,000 population (Dite 2001; Dominguez-Munoz 2014; Joergensen 2010; Spanier 2013; Yadav 2011). The prevalence of chronic pancreatitis ranges from 17 to 49 per 100,000 population (Dominguez-Munoz 2014; Joergensen 2010; Yadav 2011). The annual mortality rate attributable to chronic pancreatitis is around one to four per million people (Dominguez-Munoz 2014; Spanier 2013). Alcohol is the main cause of chronic pancreatitis (Dite 2001; Joergensen 2010; Yadav 2011). Other causes include: smoking; drugs such as valproate, thiazide, and oestrogens; other predisposing metabolic disorders and diseases such as hypercalcaemia, hyperparathyroidism, and chronic renal failure; infections such as human immunodeficiency virus (HIV) and mumps; genetic mutations such as SPINK1 or CFTR mutations; obstruction of the main pancreatic duct due to cancer, scarring post ERCP (endoscopic retrograde cholangiopancreatography) or after an attack of severe pancreatitis; recurrent pancreatitis; autoimmune pancreatitis; gallstones; and idiopathic pancreatitis (including tropical pancreatitis; Braganza 2011; Dominguez-Munoz 2014; Joergensen 2010). The reasons for these causes to result in chronic pancreatitis are poorly understood and various theories have been proposed (Braganza 2011). Increasing age and male gender are associated with a higher incidence and prevalence of chronic pancreatitis (Joergensen 2010; Spanier 2013; Yadav 2011).

While histopathological examination of a specimen of pancreas obtained by wedge biopsy or excision provides the definitive diagnosis of chronic pancreatitis, this is not practical (Braganza 2011). Invasive methods, such as reduction of bicarbonate in duodenal aspirate after stimulation with cholecystokinin or its analogue caerulein and ductal abnormalities on ERCP, are neither available routinely, nor can they be recommended routinely in patients with chronic abdominal pain (Braganza 2011). Secretin enhanced mag- netic resonance cholangiopancreatography (MRCP), endoscopic ultrasound (EUS), and computed tomography (CT) scans are the other tests that may be used for the diagnosis of chronic pancreatitis.

Various criteria have been used for the staging of chronic pancreatitis. Some of these are Ammann's criteria (Ammann 1997), the M-ANNHEIM criteria (named after the first letters of the causes of pancreatitis; Schneider 2007), the revised Japanese clinical diagnostic criteria (Shimosegawa 2010), the Manchester classification (Bagul 2006), and the Heidelberg criteria (Buchler 2009). The presence of so many classifications is clear evidence of the lack of consensus among experts about the staging of chronic pancreatitis. The validity of these different criteria, in terms of reproducibility and implications, has not been compared in order to allow the recommendation of one staging system over another. In general, the criteria for staging chronic pancreatitis include one or more of the following features: chronic abdominal pain, exocrine pancreatic insufficiency (pancreatic enzyme deficiency that leads to indigestion of food and is manifested clinically by steatorrhoea, bloating, excessive flatulence, or established by decreased stool elastase), calcifications in the pancreas, pancreatic ductal abnormalities, and histopathological diagnosis. In addition to the symptoms mentioned above, patients may also develop symptoms related to complications associated with chronic pancreatitis, such as diabetes, pancreatic pseudocysts, and biliary obstruction (Braganza 2011). Chronic abdominal pain is the major manifestation of chronic pancreatitis. The pain is usually in the upper abdomen and is usually described as deep, penetrating, and radiating to the back (Fasanella 2007). Various theories exist as to the pathogenesis of pain in patients with chronic pancreatitis. The major theories are pancreatic duct hypertension caused by calcification and fibrosis resulting from inflammation; increased pancreatic tissue pressure due to fibrosis of the peripancreatic capsule and parenchyma; and neural pain (Fasanella 2007). Due to the neural origin of pain, treatments such as pregabalin (Olesen 2011), coeliac plexus blocks and neurolysis (Puli 2009), and thoracic splanchnicectomy (division of the thoracic splanchnic nerves, which carry the sympathetic and sensory fibres from the abdominal organs including the pancreas; Bradley 2003) have been used to treat patients with chronic pancreatitis.

\section{Description of the intervention}

Pregabalin is a derivative of $\gamma$-aminobutyric acid (GABA), an inhibitory neurotransmitter (Sills 2006). Pregabalin is given orally in two or three divided doses daily (Martindale 2011). It is licensed for use for a variety of indications including: epilepsy, generalised anxiety disorder, neuropathic pain, and fibromyalgia (Martindale 2011). For neuropathic pain, the initial dose in the UK is $150 \mathrm{mg}$ daily, increased after three to seven days according to the patient's response to $300 \mathrm{mg}$ daily, and then to $600 \mathrm{mg}$ daily after another seven days. Similar doses are licensed in the USA for the treatment 
of neuropathic pain, although a maximum daily dose of $300 \mathrm{mg}$ is recommended in diabetic neuropathy (Martindale 2011).

Common adverse events related to pregabalin include: dizziness, somnolence, blurred vision, diplopia (double vision), dry mouth, constipation, vomiting, flatulence, euphoria, confusion, reduced libido, erectile dysfunction, irritability, vertigo, ataxia, tremor, dysarthria, paraesthesia, fatigue, oedema, and disturbances of attention, memory, co-ordination, and gait (Martindale 2011). Less frequent and rare but serious adverse events include: fainting episodes, heart failure, reversible renal failure, raised liver enzymes, rhabdomyolysis, breast enlargement, and gynaecomastia (Martindale 2011). Hypersensitivity reactions manifesting as rash, blisters, urticaria, dyspnoea, and wheezing have also been reported (Martindale 2011). There is currently no evidence of any metabolic interaction of pregabalin with other drugs.

\section{How the intervention might work}

Pregabalin inhibits the transmission of pain through the nerves. However, the molecular mechanism of its action is not known. Inhibition of calcium flow through high-voltage-gated calcium channels containing the $\alpha_{2} \delta$-1 subunit, resulting in decreased neurotransmitter release and excitability of the pain nerve fibres, is currently believed to be the mechanism of its action (Sills 2006).

\section{Why it is important to do this review}

There is currently no consensus on the management of pain in patients with chronic pancreatitis. This review provides the best level of evidence on the benefits and harms of pregabalin, and so allow patients and the surgeons involved in their care to make informed decisions.

\section{O B J E C T I VES}

To assess the benefits and harms of pregabalin in people with chronic pancreatitis with chronic abdominal pain.

\section{METHODS}

\section{Criteria for considering studies for this review}

\section{Types of studies}

We included randomised controlled trials (RCTs). We included studies reported in full text, those published as an abstract only, and unpublished data.

\section{Types of participants}

We included adults with chronic pancreatitis with chronic abdominal pain. We excluded patients with pancreatic cancer.

\section{Types of interventions}

We included trials comparing pregabalin (irrespective of the dose) with an inactive intervention (placebo or no intervention). We accepted co-interventions, for example, the use of a pancreatic enzyme supplement or opiates, provided that they were used equally in both groups.

\section{Types of outcome measures}

\section{Primary outcomes}

1. Health-related quality of life post-treatment (using any validated scale, such as EQ-5D or SF-36; EuroQol 2014; Ware 2014). The EQ-5D assesses the quality of life under five domains: mobility, self care, usual activities, pain or discomfort, and anxiety or depression (EuroQol 2014). The SF-36 assesses the quality of life under eight sections: vitality, physical functioning, bodily pain, general health perceptions, physical role functioning, emotional role functioning, social role functioning, and mental health (Ware 2014).

i) Short-term (two weeks to three months).

ii) Medium-term (three months to five years).

iii) Long-term (more than five years).

2. Treatment-related serious adverse events such as heart or renal failure (within three months). We accepted the following definitions of serious adverse events.

i) ICH-GCP International Conference on Harmonisation - Good Clinical Practice guideline (ICH-GCP 1996): serious adverse events defined as any untoward medical occurrence that results in death, is life-threatening, requires inpatient hospitalisation or prolongation of existing hospitalisation, or results in persistent or significant disability or incapacity.

ii) Other variations of ICH-GCP classifications such as the US Food and Drug Administration (FDA) classification (FDA 2006), or the Medicines and Healthcare products Regulatory Agency (MHRA) classification (MHRA 2013).

3. Opiate requirement (in terms of the total amount required during the follow-up period, and only from trials where opiates were not used routinely).

i) Short-term (two weeks to three months).

ii) Medium-term (three months to five years).

iii) Long-term (more than five years).

Pregabalin for decreasing pancreatic pain in chronic pancreatitis (Review) 


\section{Secondary outcomes}

1. Treatment-related adverse events (within three months; dizziness, dry mouth). We accepted all adverse events reported by the study author, irrespective of the severity of the adverse event.

2. Pain scores using a visual analogue scale.

i) Short-term (two weeks to three months).

ii) Medium-term (three months to five years).

iii) Long-term (more than five years).

3. Work days lost.

4. Number and duration of hospital admissions for control of pain.

The choice of the above clinical outcomes was based on the necessity to assess whether pregabalin is safe and effective in terms of decreasing pain and improving health-related quality of life in patients with pancreatic pain. This information would provide the data to assess the cost-effectiveness of pregabalin.

Reporting of the outcomes listed here was not an inclusion criterion for the review.

\section{Search methods for identification of studies}

\section{Electronic searches}

We conducted a literature search to identify all published and unpublished randomised controlled trials. The literature search identified potential studies in all languages. We had planned to translate the non-English language papers and fully assess them for potential inclusion in the review as necessary.

We searched the following electronic databases for identifying potential studies:

- Cochrane Central Register of Controlled Trials

(CENTRAL; The Cochrane Library 2015, Issue 6; Appendix 2);

- MEDLINE (1966 to June 2015; Appendix 3);

- EMBASE (1988 to June 2015; Appendix 4); and

- Science Citation Index (1982 to June 2015; Appendix 5).

We also conducted a search of ClinicalTrials.gov (Appendix 6), and WHO ICTRP (World Health Organization - International Clinical Trials Registry Platform; Appendix 7).

\section{Searching other resources}

We checked the reference lists of all primary studies and review articles for additional references. We attempted to contact the authors of identified trials and ask them to identify other published and unpublished studies.

We searched for errata or retractions from eligible trials on PubMed on 23 June 2015.

\section{Data collection and analysis}

\section{Selection of studies}

Two review authors (KG and $\mathrm{CL}$ ) independently screened the titles and abstracts of all the potential studies we identified as a result of the search and coded them as 'retrieve' (eligible, potentially eligible, or unclear) or 'do not retrieve'. We retrieved the full-text study reports and two review authors (KG and CL) independently screened the full text, identified studies for inclusion, and identified and recorded the reasons for exclusion of the ineligible studies. We resolved any disagreement through discussion. We identified and excluded duplicate references and collated multiple reports of the same study so that each study rather than each report was the unit of interest in the review. We recorded the selection process in sufficient detail to complete a PRISMA flow diagram and 'Characteristics of excluded studies' table.

\section{Data extraction and management}

We used a standard data collection form for study characteristics and outcome data. Two review authors (KG and CL) extracted the following study characteristics from the included studies:

1. Methods: study design, total duration study and run-in, number of study centres and location, study setting, withdrawals, date of study.

2. Participants: number $(\mathrm{N})$, mean age, age range, gender, inclusion criteria, exclusion criteria.

3. Interventions: intervention, comparison, concomitant interventions.

4. Outcomes: primary and secondary outcomes specified and collected, time points reported.

5. Notes: funding for trial, notable conflicts of interest of trial authors.

Two review authors (KG and CL) independently extracted outcome data from the included studies. If outcomes were reported at multiple time points, for example, short-term health-related quality of life was reported at two weeks and three weeks, we chose the later time point (i.e. three weeks) for data extraction. If possible, for time-to-event outcomes, we had planned to extract data to calculate the natural logarithm of the hazard ratio and its standard error, using the methods suggested by Parmar, et al (Parmar 1998). We had planned to include all randomised participants for medium-term and long-term outcomes (for example, quality of life); this would not have been conditional upon the short-term outcomes (for example, having a low- or high-quality of life index at three weeks).

We had planned to note in the 'Characteristics of included studies' table if outcome data were reported in an unusable way. We resolved disagreements by consensus. One review author (KG) copied the data from the data collection form into the Review Manager file. We double checked that the data were entered cor- 
rectly by comparing the study reports with the data presentation in the review.

\section{Assessment of risk of bias in included studies}

Two review authors (KG and CL) independently assessed the risk of bias for each study using the criteria outlined in the Cochrane Handbook for Systematic Reviews of Interventions (Higgins 2011). We resolved any disagreement by discussion. We assessed the risk of bias according to the following domains:

1. random sequence generation;

2. allocation concealment;

3. blinding of participants and personnel;

4. blinding of outcome assessment;

5. incomplete outcome data;

6. selective outcome reporting;

7. other bias.

We graded each potential source of bias as high, low, or unclear, and provided a quote from the study report, together with a justification for our judgement in the 'Risk of bias' table. We summarised the 'Risk of bias' judgements across different studies for each of the domains listed. We considered blinding separately for different key outcomes where necessary. Where information on risk of bias related to unpublished data or correspondence with a trialist, we had planned to note this in the 'Risk of bias' table. When considering treatment effects, we took into account the risk of bias of the studies that contributed to that outcome.

\section{Assessment of bias in conducting the systematic review}

We conducted the review according to the published protocol and reported any deviations from it in the 'Differences between protocol and review' section of the systematic review.

\section{Measures of treatment effect}

We analysed dichotomous data (proportion of participants with adverse events, serious adverse events, and number of hospital admissions) as a risk ratio. We had planned to analyse continuous data as a mean difference when the outcome was reported in the same units (pain scores, number of work days lost, and duration of hospital admissions for control of pain), or the same health-related quality of life scale; or as a standardised mean difference when different scales were used for measuring quality of life, or when different opiates were used at different doses. We have ensured that higher scores for continuous outcomes have the same meaning for the particular outcome, explained the direction to the reader, and reported where the directions were reversed, if this was necessary. We had planned to calculate the rate ratio for outcomes such as adverse events and serious adverse events, where it was possible for the same person to develop more than one adverse event (or serious adverse event). If the authors had calculated the rate ratio of adverse events (or serious adverse events) in the intervention versus the control group based on Poisson regression, we had planned to obtain the rate ratio by the Poisson regression method in preference to the rate ratio calculated based on the number of adverse events (or serious adverse events) during a certain period. We had planned to calculate the hazard ratio for time-to-event outcomes such as time-to-first adverse event (or serious adverse event).

We had planned to undertake meta-analyses only where this was meaningful, i.e. if the treatments, participants, and the underlying clinical question were similar enough for pooling to make sense.

A common way in which trialists indicate that they have skewed data is by reporting medians and interquartile ranges. When we encountered this, we had planned to note that the data were skewed, and consider the implications of this.

Where multiple trial arms were reported in a single trial, we had planned to include only the relevant arms. If two comparisons had to be entered into the same meta-analysis (e.g. pregabalin low dose versus placebo and pregabalin high dose versus placebo), we had planned to halve the control group to avoid double counting. The alternative way of including trials with multiple arms is to pool the results of the pregabalin doses and compare this with placebo. We had planned to perform a sensitivity analysis to determine if the results of the two methods of dealing with multi-arm trials led to different conclusions.

\section{Unit of analysis issues}

The unit of analysis was individual patients with chronic pancreatitis with abdominal pain. We did not anticipate any clusterrandomised trials for this comparison, but if cluster-randomised trials were identified, we had planned to obtain the effect estimate, adjusted for the clustering effect. If this was not available, we had planned to perform a sensitivity analysis by excluding the trial from the meta-analysis, as the variance of the effect estimate unadjusted for the cluster effect is less than the actual variance, which is adjusted for the cluster effect giving inappropriately more weight to the cluster-RCT in the meta-analysis.

Had we included cross-over randomised trials, we had planned to include only the data prior to cross-over, and analyse the data in the same way as parallel randomised trials.

\section{Dealing with missing data}

We would have attempted to contact investigators or sponsors in order to verify key study characteristics and obtain missing numerical outcome data when indicated (e.g. when a study was identified as an abstract only). If we were unable to obtain the information from the investigators or study sponsors, we had planned to impute the mean from the median (i.e. consider the median as the mean) and the standard deviation from the standard error or P values, according to the Cochrane Handbook for Systematic Reviews of Interventions (Higgins 2011); we had planned to assess the impact of including such studies in a sensitivity analysis, as 
indicated. If we were unable to calculate the standard deviation from the standard error or P values, we had planned to impute the standard deviation as the highest standard deviation in the remaining trials included in the outcome, fully aware that this method of imputation would decrease the weight of the studies in the metaanalysis of mean difference, and shift the effect towards no effect for the standardised mean difference.

\section{Assessment of heterogeneity}

We planned to use the $\mathrm{I}^{2}$ statistic to measure heterogeneity among the trials in each analysis. If we identified substantial heterogeneity, as per the Cochrane Handbook for Systematic Reviews of Interventions (greater than 50\% to 60\%; Higgins 2011), we had planned to explore it by pre-specified subgroup analysis. We had also planned to assess heterogeneity by evaluating whether there was good overlap of confidence intervals.

\section{Assessment of reporting biases}

We would have attempted to contact study authors to ask them to provide missing outcome data. Where this was not possible, and the missing data were thought to introduce serious bias, we had planned to explore the impact of including such studies in the overall assessment of results by a sensitivity analysis.

If we had been able to pool more than 10 trials, we had planned to create and examine a funnel plot to explore possible publication biases. We had planned to use Egger's test to determine the statistical significance of the reporting bias (Egger 1997). We had planned to consider a P value of less than 0.05 to be a statistically significant reporting bias.

\section{Data synthesis}

We performed the analysis using RevMan 5.3 (Review Manager 2014). We had planned to use the Mantel-Haenszel method for dichotomous data, the inverse variance method for continuous data, and the generic inverse variance method for count and timeto-event data. We had planned to use both the fixed-effect model (Demets 1987), and the random-effects model (DerSimonian 1986), for the analysis. In case of discrepancy between the two models, we had planned to report both results; otherwise we had planned to report only the results from the fixed-effect model.

\section{'Summary of findings' table}

We created a 'Summary of findings' table using all of the outcomes. We used the five GRADE considerations (study limitations, consistency of effect, imprecision, indirectness, and publication bias) to assess the quality of the body of evidence as it related to the studies that contributed data to the meta-analyses for the pre-specified outcomes. We used the methods and recommendations described in Chapter 8.5 and Chapter 12 of the Cochrane Handbook for Systematic Reviews of Interventions (Higgins 2011), and used GRADEpro software. We justified all decisions to downgrade or upgrade the quality of studies using footnotes, and made comments to aid the reader's understanding of the review where necessary. We had planned to consider whether there was any additional outcome information that could not be incorporated into the meta-analyses, note this in the comments, and state if it supported or contradicted the information from the meta-analyses.

\section{Subgroup analysis and investigation of heterogeneity}

We had planned to carry out the following subgroup analyses:

1. Different doses of pregabalin (each different dose of pregabalin).

2. Routine pancreatic enzyme supplementation versus no pancreatic enzyme supplementation.

3. Participants in whom opiates were used routinely versus participants in whom opiates were not used routinely.

We had planned to use all of the primary outcomes in subgroup analyses (except for the outcome opiate requirement for the subgroup analysis of routine opiate use versus no routine opiate use). We had planned to use the formal $\mathrm{Chi}^{2}$ test for subgroup differences to test for subgroup interactions.

\section{Sensitivity analysis}

We had planned to perform the following sensitivity analysis, defined a priori, to assess the robustness of our conclusions:

1. exclude trials at unclear or high risk of bias (one of more of the 'Risk of bias' domains (other than blinding of surgeon) classified as unclear or high);

2. exclude trials in which either the mean, standard deviation, or both were imputed;

3. exclude cluster-RCTs in which the adjusted effect estimates are not reported;

4. explore different methods of dealing with multi-arm trials (please see Measures of treatment effect).

\section{Reaching conclusions}

We based our conclusions on the findings from the quantitative and narrative synthesis of the included studies in this review. We avoided making recommendations for practice; our implications for research have given the reader a clear sense of where the focus of future research in the area should be and what the remaining uncertainties are.

\section{RES U L T S}

\section{Description of studies}




\section{Results of the search}

We identified 112 references through electronic searches of CENTRAL (Wiley) $(\mathrm{N}=6)$, MEDLINE (OvidSP) $(\mathrm{N}=13)$, EMBASE (OvidSP) $(\mathrm{N}=76)$, Science Citation Index expanded $(\mathrm{N}$ =11), ClinicalTrials.gov $(\mathrm{N}=1)$, and WHO Trials register $(\mathrm{N}=$ 5). After removing duplicate references, there were 82 references. We excluded 73 clearly irrelevant references through reading titles and abstracts. A total of nine references were retrieved for further assessment from the full publication. All the nine references were eligible for inclusion in the review. All nine references were reports of the same randomised controlled trial (Olesen 2011), which fulfilled the inclusion criteria (Characteristics of included studies). The reference flow is shown in Figure 1. 
Figure I. Study flow diagram.

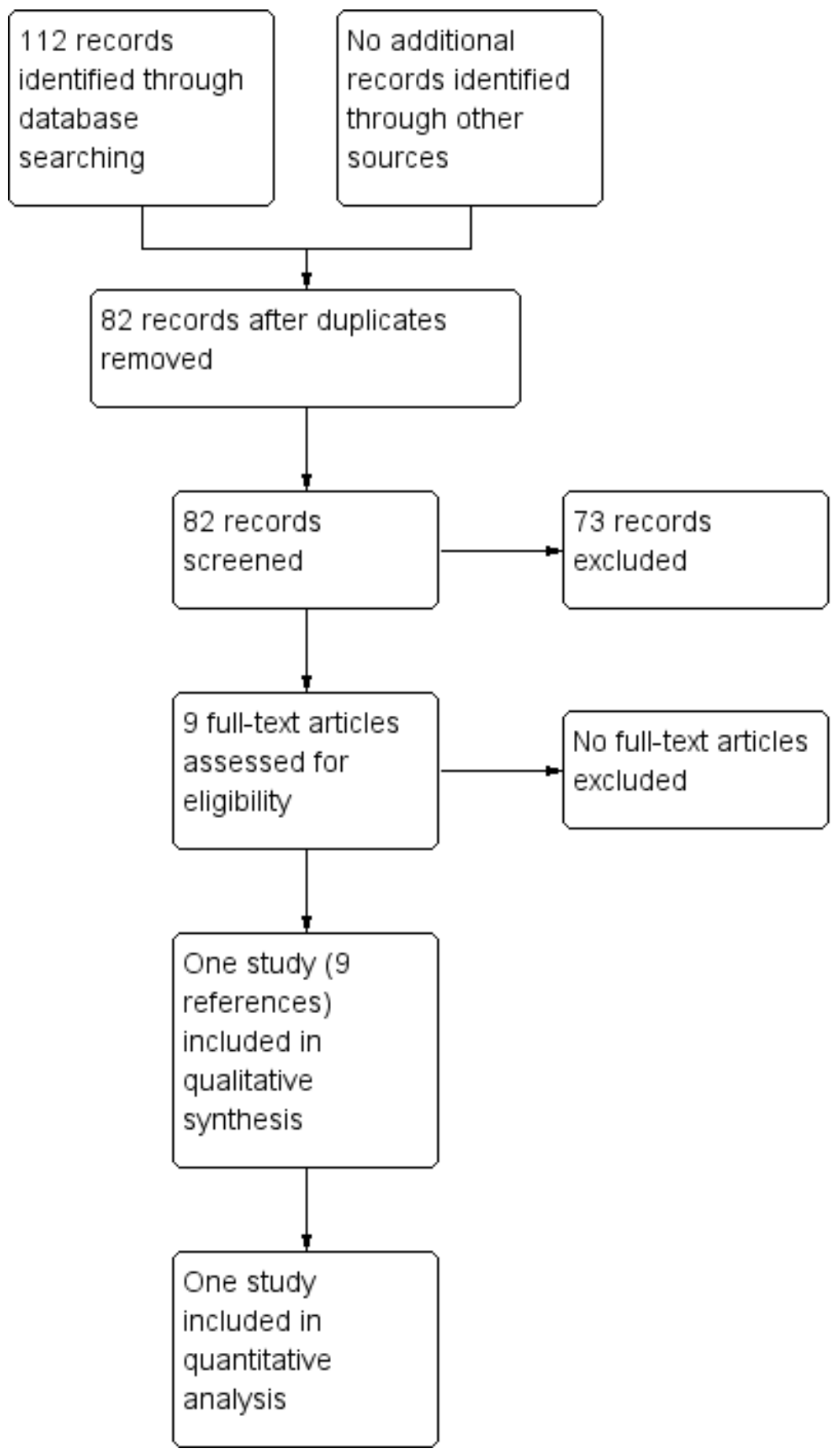




\section{Included studies}

One randomised controlled trial (RCT) was included in this review (Olesen 2011). This was a two-armed trial in which 64 participants with chronic pancreatitis and pancreatic pain were randomised to escalating doses of pregabalin $(150 \mathrm{mg}$ per day to 600 mg per day; 34 participants) or matching placebo (30 participants). Participants received pregabalin or placebo for three weeks on an outpatient basis; the outcomes were measured at the end of the treatment (i.e. three weeks from commencement of treatment). Potential participants taking concomitant analgesic medication and expected to stay on a stable regime during the trial were allowed to enter the study. There was no evidence of any baseline differences in the pain scores or analgesic use between the groups. The proportion of participants with obstructive jaundice was not stated in the trial. The outcomes of interest for this review that were reported in the trial included: health-related quality of life, opiate analgesic requirement, adverse events and serious adverse events, pain scores, and hospital readmissions. The trial also reported several other surrogate outcomes that explored the mechanism of action of pregabalin (Olesen 2011).

\section{Excluded studies}

We did not exclude any reference after obtaining the full text, since it was clear that none of the references met the inclusion criteria on reading the titles and abstracts.

\section{Risk of bias in included studies}

The only trial included in this review was a trial at a low risk of bias. The risk of bias in individual domains is shown in Figure 2. 
Figure 2. Risk of bias summary: review authors' judgements about each 'Risk of bias' item for each included study.

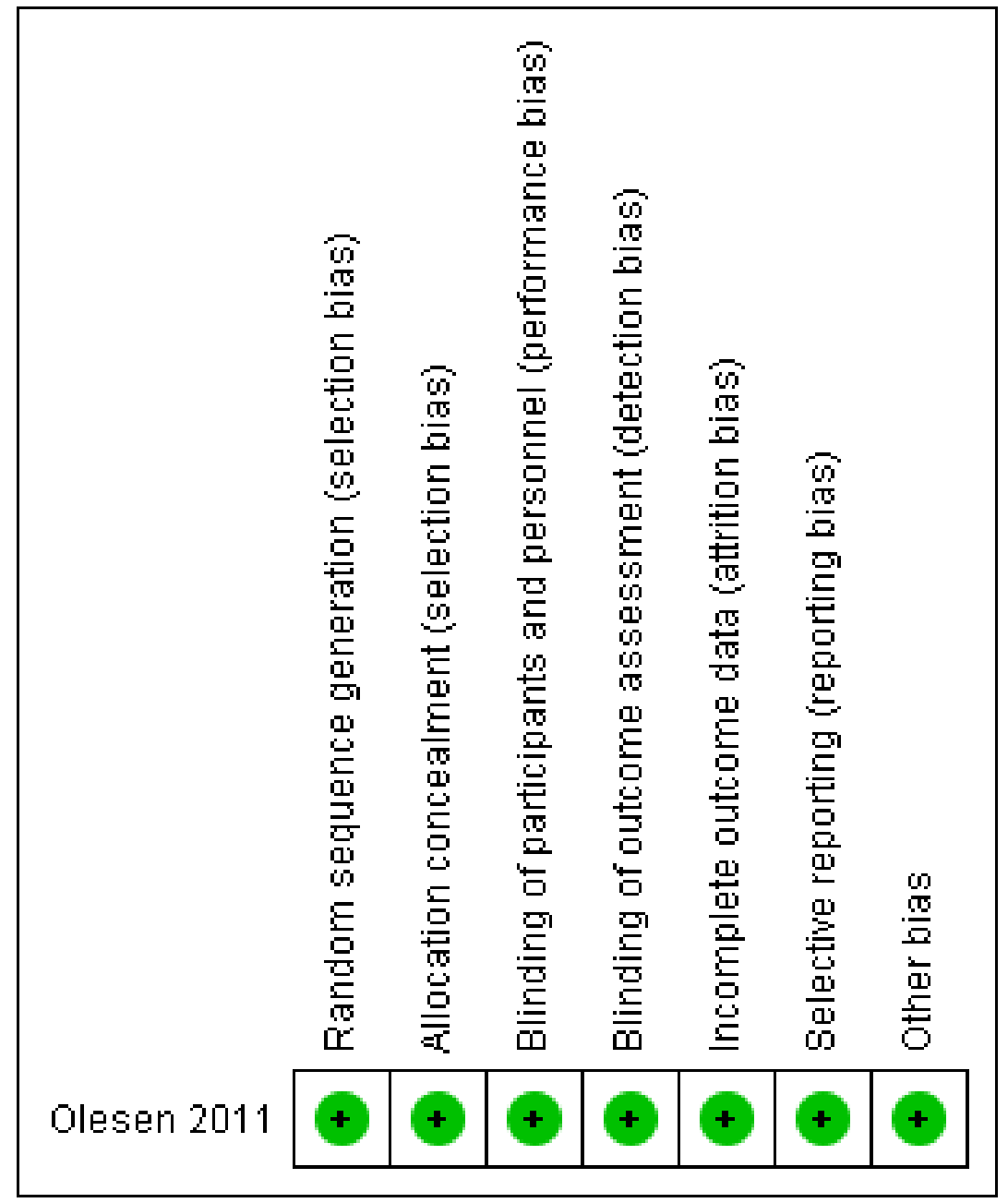

\section{Allocation}

The random sequence was generated by computer and was concealed from researchers by the sponsor.

\section{Blinding}

Participants, healthcare providers, and outcome assessors were blinded to the use of placebo.

\section{Incomplete outcome data}

All the participants were included in the analyses, and an intentionto-treat analysis was performed.

\section{Selective reporting}

The trial reported the important short-term outcomes that could be expected to be reported.

\section{Other potential sources of bias}

We did not identify any other potential sources of bias in the trial. However, it has to be noted that the trial was funded by a party with a vested interest in the results.

\section{Effects of interventions}

See: Summary of findings for the main comparison Pregabalin versus placebo for decreasing pancreatic pain in chronic 
pancreatitis

The only trial included in this review followed 64 participants for three weeks after commencement of treatment (Olesen 2011). So, only the short-term outcomes were available. The medium and long-term outcomes were not available from this trial. The trial did not report the number of work days lost and length of hospital stay (Olesen 2011). Since there was only trial, we did not assess heterogeneity, perform meta-analysis using fixed-effect or random-effects model, assess reporting bias, or perform subgroup or sensitivity analysis. A summary of the effect estimates is available in the Summary of findings for the main comparison.

\section{Primary outcomes}

\section{Health-related quality of life (short-term)}

The trial reported change in health-related quality of life (HRQoL; measured using EORTC CLQ-30 questionnaire) from the baseline. This is a scale of 0 to 100 with higher values indicating a better HRQoL.There was no statistically significant difference in the change in HRQoL at three weeks favouring the pregabalin group (MD 11.40; 95\% CI -3.28 to 26.08; participants = 64; studies = 1; Analysis 1.1). It should be noted that standard deviation was calculated from confidence intervals reported by the authors.

\section{Serious adverse events}

There was no statistically significant difference in the proportion of people who developed serious adverse events between the two groups (RR 1.76; 95\% CI 0.35 to 8.96; participants = 64; studies $=1 ;$ Analysis 1.2 ).

\section{Opiate requirement (short-term)}

The trial reported a change in opiate requirement (measured in $\mathrm{mg}$ but the opiate used was not reported) from the baseline. There was a statistically significant difference in the reduction in opiate use at three weeks favouring the pregabalin group (MD $-26.00 \mathrm{mg}$; 95\% CI -47.36 to -4.64 ; participants = 64; studies = 1; Analysis 1.3). It should be noted that the standard deviation was calculated from the $\mathrm{P}$ value reported by the authors.

\section{Secondary outcomes}

\section{Adverse events}

The proportion of people with adverse events was statistically significantly more in the pregabalin group than placebo group (RR $1.71 ; 95 \%$ CI 1.20 to 2.43; participants $=64$; studies $=1$; Analysis 1.4 .

\section{Pain scores (short-term)}

The trial reported percentage change in pain scores (measured using visual analogue scale) from the baseline. There was a statistically significant difference in the percentage change in pain scores at three weeks favouring the pregabalin group (MD -12.00; $95 \%$ CI -21.82 to -2.18 ; participants $=64$; studies $=1$; Analysis 1.5$)$. It should be noted that the standard deviation was calculated from the confidence intervals reported by the authors.

\section{Hospital admissions for control of pain}

There was no statistically significant difference in the proportion of people who required hospital admissions for pain control between the two groups (RR 0.44; 95\% CI 0.04 to 4.62; participants = 64; studies = 1; Analysis 1.6.

\section{DISCUSSION}

\section{Summary of main results}

We only identified one randomised controlled trial assessing the effect of pregabalin in decreasing pancreatic pain in chronic pancreatitis (Olesen 2011). This trial found that opiate use and pain scores were lower in participants taking pregabalin compared to those taking placebo. This trial also found that there were more adverse events in participants taking pregabalin compared to those taking placebo. There was no statistically significant difference in the short-term health-related quality of life, proportion of people with serious adverse events, or in hospital admissions. The trial did not assess medium and long-term outcomes, number of work days lost, or length of hospital stay.

\section{Overall completeness and applicability of evidence}

The trial only included patients with chronic pain due to chronic pancreatitis, so the results are only applicable for people with these diagnoses and symptoms. The benefits and harms of short-term administration of pregabalin was reported, but these cannot be extrapolated to benefits and harms in the medium- and long-term, which may be entirely different from those in the short-term.

\section{Quality of the evidence}

The overall quality of the evidence was moderate or low. The risk of bias was low in the trial. The major issue was the duration of the trial and follow-up (i.e. medium to long-term outcomes were

Pregabalin for decreasing pancreatic pain in chronic pancreatitis (Review) 
not available), and the lack of important clinical outcomes, such as work days lost and length of hospital stay.

\section{Potential biases in the review process}

We performed a thorough literature search using formal search strategies. At least two review authors independently identified trials for inclusion and extracted data, thus minimising errors in these aspects. We were unable to explore publication bias because of the presence of only one trial in this review. However, we searched the trial registers, which revealed only one trial on this topic. Pregabalin is a relatively new discovery and we anticipate that trials related to this topic are registered prospectively, so, the risk of reporting bias is small (Martindale 2011).

\section{Agreements and disagreements with other studies or reviews}

This is the first review on this topic. We disagree with the trial authors that pregabalin is an effective adjuvant therapy for treatment of pain due to chronic pancreatitis because of the lack of information on medium-term and long-term outcomes, and lack of evidence on clinical benefits.

Chronic pancreatitis, as the name indicates, is a long-standing disorder, with few deaths attributed to it (one to four per million people (Dominguez-Munoz 2014; Spanier 2013)). However, it might have a significant socioeconomic impact due to hospital admissions and loss of work days due to chronic pain (Hall 2014). A steady state of pregabalin levels can be reached in one to two days (Martindale 2011), and one can expect the drug to have an effect in one or two days (Gajraj 2007). Pregabalin has a relatively short half-life, with a mean elimination half-life of 6.3 hours (Martindale 2011). Based on this half-life, it is reasonable to expect the actions of pregabalin to only last as long as it is being taken.

The only trial assessing the effect of pregabalin on patients with chronic pain due to chronic pancreatitis administered pregabalin to the participants for a period of three weeks. This period is not sufficient to evaluate the beneficial and harmful effect of pregabalin, since clinically, a patient might be required to take pregabalin for a prolonged period of time for pain control. The clinical significance of a change of short-term health-related quality of life, opiate use, and pain scores from baseline was not evident, since the trial was not powered to measure differences in clinical outcomes, such as hospital admissions, and did not report on the work days lost because of pain.

Participants taking pregabalin reported more adverse events than those taking placebo, although the vast majority of these were mild (Olesen 2011). Common adverse events related to pregabalin include: dizziness, somnolence, blurred vision, diplopia (double vision), dry mouth, constipation, vomiting, flatulence, euphoria, confusion, reduced libido, erectile dysfunction, irritability, vertigo, ataxia, tremor, dysarthria, paraesthesia, fatigue, oedema, and disturbances of attention, memory, co-ordination, and gait (Martindale 2011). These adverse events cannot be ignored. While patients may develop tolerance to some adverse events, they may not develop tolerance for others, such as somnolence (Kanbayashi 2014).

Thus, based on the lack of information on medium-term and longterm outcomes, and lack of evidence on clinical benefits, pregabalin cannot be routinely recommended in patients with chronic pain due to chronic pancreatitis. However, there is a need to conduct further randomised controlled trials on this topic because of the socioeconomic impact of chronic pancreatitis. Such trials should be powered to measure clinically relevant pain scores and quality of life. For example, a trial comparing early surgery with medical treatment followed by endoscopic treatment and surgical treatment (step-up approach) for chronic pancreatitis used a minimum difference of 15 points on a visual analogue scale of 0 to 100 , based on the results of a consensus meeting of an expert panel (Ahmed Ali 2013). The trial authors estimated that a trial would require approximately 88 participants to identify this minimal clinically important difference, based on an alpha of 0.05 and power of 0.90 (Ahmed Ali 2013). Given the nature of the intervention, it is possible to conduct a trial at low risk of bias. Such a trial should assess the medium or long-term effects of pregabalin and should include outcomes such as: quality of life, treatment-related adverse events, number of work days lost, number of hospital admissions, and the length of hospital stay, to assess the clinical and socioeconomic impact of using pregabalin in patients with chronic pain due to chronic pancreatitis.

\section{A U THORS'CONCLUSIONS}

\section{Implications for practice}

Based on low- to moderate-quality evidence, short-term pregabalin decreases short-term opiate use, and short-term pain scores, but increases adverse events compared to placebo, in people with chronic pain due to chronic pancreatitis. The clinical implication of reduced short-term opiate use and pain scores is not known.

\section{Implications for research}

Future trials assessing the role of pregabalin in decreasing pain in chronic pancreatitis should assess the medium or long-term effects of pregabalin, and should include outcomes such as: quality of life, treatment-related adverse events, number of work days lost, number of hospital admissions, and the length of hospital stay, in addition to pain scores, to assess the clinical and socioeconomic impact. 


\section{ACKNOWLEDGEMENTS}

We thank Karin Dearness, Managing Editor, Cochrane Upper Gastrointestinal and Pancreatic Diseases (UGPD) Group, for providing administrative and logistical support for the conduct of the current review, and Racquel Simpson, Trials Search Co-ordinator, Cochrane Upper Gastrointestinal and Pancreatic Diseases (UGPD) Group, for developing and executing the search strategies.

We thank the copy editors and Cochrane Editorial Unit for their comments.

\section{REFEREN CES}

\section{References to studies included in this review}

\section{Olesen 2011 \{published data only\}}

Bouwense SA, Olesen SS, Drewes AM, Poley JW, Goor $\mathrm{H}$, Wilder-Smith OH. Effects of pregabalin on central sensitization in patients with chronic pancreatitis in a randomized, controlled trial. PLoS One 2012;7(8):e42096. [DOI: 10.1371/journal.pone.0042096] Bouwense SAW, Olesen SS, Drewes AM, Poley JW, Van Goor H, Wilder-Smith OHG. Pregabalin reduces central sensitisation in chronic pancreatitis. European Journal of Pain Supplements 2011;5(1):21.

Graversen C, Olesen SS, Olesen AE, Drewes AM. The analgesic effect of pregabalin is correlated to alterations in pharmaco-EEG in chronic pain patients. European Journal of Pain Supplements 2011;5(1):113.

Graversen C, Olesen SS, Olesen AE, Steimle K, Farina D, Wilder-Smith $\mathrm{OH}$, et al. The analgesic effect of pregabalin in patients with chronic pain is reflected by changes in pharmaco-EEG spectral indices. British Journal of Clinical Pharmacology 2012;73(3):363-72.

Malver LP, Olesen SS, Graversen C, Olesen AE, Frokjaer $\mathrm{JB}$, Wilder-Smith $\mathrm{O}$, et al. Effect of pregabalin on visceral sensation and central pain processing in patients with chronic pancreatitis. European Journal of Pain Supplements 2011;5(1):277.

* Olesen SS, Bouwense SA, Wilder-Smith OH, Goor $\mathrm{H}$, Drewes AM. Pregabalin reduces pain in patients with chronic pancreatitis in a randomized, controlled trial. Gastroenterology 2011;141(2):536-43.

Olesen SS, Bouwense SA, Wilder-Smith OH, Van Goor $\mathrm{H}$, Drewes AM. Pregabalin for pain treatment in chronic pancreatitis. Gastroenterology 2011;140(5 Suppl):S68-S9. Olesen SS, Graversen C, Bouwense SAW, van Goor H, Wilder-Smith OHG, Drewes AM. Quantitative sensory testing predicts pregabalin efficacy in painful chronic pancreatitis. PLoS One 2013;8(3):e57963.

Olesen SS, Graversen C, Olesen AE, Frøkjaer JB, WilderSmith $\mathrm{O}$, Goor $\mathrm{H}$, et al. Randomised clinical trial: Pregabalin attenuates experimental visceral pain through sub-cortical mechanisms in patients with painful chronic pancreatitis. Alimentary Pharmacology and Therapeutics 2011;34(8):878-87.

\section{Additional references}

\section{Ahmed Ali 2013}

Ahmed Ali U, Issa Y, Bruno MJ, van Goor H, van Santvoort $\mathrm{H}$, Busch OR, et al. Early surgery versus optimal current step-up practice for chronic pancreatitis (ESCAPE): design and rationale of a randomized trial. BMC Gastroenterology 2013;13:49.

\section{Ammann 1997}

Ammann RW. A clinically based classification system for alcoholic chronic pancreatitis: summary of an international workshop on chronic pancreatitis. Pancreas 1997;14(3): 215-21.

Bagul 2006

Bagul A, Siriwardena AK. Evaluation of the Manchester classification system for chronic pancreatitis. Journal of the Pancreas 2006;7(4):390-6.

Bradley 2003

Bradley 3rd EL, Bem J. Nerve blocks and neuroablative surgery for chronic pancreatitis. World Journal of Surgery 2003;27(11):1241-8.

\section{Braganza 2011}

Braganza JM, Lee SH, McCloy RF, McMahon MJ. Chronic pancreatitis. Lancet 2011;377(9772):1184-97.

\section{Buchler 2009}

Buchler MW, Martignoni ME, Friess H, Malfertheiner P. A proposal for a new clinical classification of chronic pancreatitis. BMC Gastroenterology 2009;9:93.

\section{Demets 1987}

Demets DL. Methods for combining randomized clinical trials: strengths and limitations. Statistics in Medicine 1987; 6(3):341-50.

\section{DerSimonian 1986}

DerSimonian R, Laird N. Meta-analysis in clinical trials. Controlled Clinical Trials 1986;7(3):177-88. 
Dite 2001

Dite P, Stary K, Novotny I, Precechtelova M, Dolina J, Lata J, et al. Incidence of chronic pancreatitis in the Czech Republic. European Journal of Gastroenterology and Hepatology 2001;13(6):749-50.

Dominguez-Munoz 2014

Dominguez-Munoz JE, Lucendo A, Carballo LF, IglesiasGarcia J, Tenias JM. A Spanish multicenter study to estimate the prevalence and incidence of chronic pancreatitis and its complications. Revista Española de Enfermedades Digestivas 2014;106(4):239-45.

Egger 1997

Egger M, Davey SG, Schneider M, Minder C. Bias in metaanalysis detected by a simple, graphical test. BMJ (Clinical Research Ed.) 1997;315(7109):629-34.

\section{EuroQol 2014}

EuroQol. About EQ-5D. http://www.euroqol.org/abouteq-5d.html (accessed 8 October 2014).

\section{Fasanella 2007}

Fasanella KE, Davis B, Lyons J, Chen Z, Lee KK, Slivka A, et al. Pain in chronic pancreatitis and pancreatic cancer. Gastroenterology Clinics of North America 2007;36(2):33564, ix.

\section{FDA 2006}

Center for Biologics Evaluation and Research, U.S. Food, Drug Administration. Guidance for industry adverse reactions section of labelling for human prescription drug and biological products - Content and format. http://www.fda.gov/downloads/Drugs/ GuidanceComplianceRegulatoryInformation/Guidances/ ucm075057.pdf 2006 (accessed 4 July 2014).

\section{Gajraj 2007}

Gajraj NM. Pregabalin: its pharmacology and use in pain management. Anesthesia and Analgesia 2007;105(6): 1805-15.

\section{Hall 2014}

Hall TC, Garcea G, Webb MA, Al-Leswas D, Metcalfe MS, Dennison AR. The socio-economic impact of chronic pancreatitis: a systematic review. Journal of Evaluation in Clinical Practice 2014;20(3):203-7.

\section{Higgins 2011}

Higgins JPT, Green S (editors). Cochrane Handbook for Systematic Reviews of Interventions Version 5.1.0 [updated March 2011]. The Cochrane Collaboration, 2011. Available from www.cochrane-handbook.org.

\section{ICH-GCP 1996}

International Conference on Harmonisation of Technical Requirements for Registration of Pharmaceuticals for Human Use. Code of Federal Regulation \& ICH Guidelines. Media: Parexel Barnett, 1996.

Joergensen 2010

Joergensen M, Brusgaard K, Cruger DG, Gerdes AM, de Muckadell OB. Incidence, prevalence, etiology, and prognosis of first-time chronic pancreatitis in young patients: a nationwide cohort study. Digestive Diseases and Sciences 2010;55(10):2988-98.

\section{Kanbayashi 2014}

Kanbayashi Y, Onishi K, Hosokawa T. Factors predicting adverse events associated with pregabalin administered for neuropathic pain relief. Pain research \& management : the journal of the Canadian Pain Society = journal de la societe canadienne pour le traitement de la douleur 2014;19(6): e164-7.

\section{Martindale 2011}

Sweetman S (editor). Martindale: the complete drug reference (online version), 37th edition. www.pharmpress.com/product/MC MART/martindalethe-complete-drug-reference 2011 (accessed 23 September 2014).

\section{MHRA 2013}

Medicines and Healthcare products Regulatory Agency (MHRA). Clinical trials for medicines: Safety reporting - SUSARs and DSURs. http://www.mhra.gov.uk/ Howweregulate/Medicines/Licensingofmedicines/ Clinicaltrials/Safetyreporting-SUSARsandASRs/ 2013 (accessed 4 July 2014).

\section{NCBI 2014}

NCBI. MeSH. NLM Controlled Vocabulary. Pancreas. http://www.ncbi.nlm.nih.gov/mesh/68010179 2014 (accessed 4 July 2014).

\section{Parmar 1998}

Parmar MK, Torri V, Stewart L. Extracting summary statistics to perform meta-analyses of the published literature for survival endpoints. Statistics in Medicine 1998;17(24): 2815-34.

\section{Puli 2009}

Puli SR, Reddy JB, Bechtold ML, Antillon MR, Brugge WR. EUS-guided celiac plexus neurolysis for pain due to chronic pancreatitis or pancreatic cancer pain: a metaanalysis and systematic review. Digestive Diseases and Sciences 2009;54(11):2330-7.

\section{Review Manager 2014 [Computer program]}

The Nordic Cochrane Centre, The Cochrane Collaboration. Review Manager (RevMan). Version 5.3. Copenhagen: The Nordic Cochrane Centre, The Cochrane Collaboration, 2014.

Schneider 2007

Schneider A, Lohr JM, Singer MV. The M-ANNHEIM classification of chronic pancreatitis: introduction of a unifying classification system based on a review of previous classifications of the disease. Journal of Gastroenterology 2007;42(2):101-19.

\section{Shimosegawa 2010}

Shimosegawa T, Kataoka K, Kamisawa T, Miyakawa $\mathrm{H}$, Ohara $\mathrm{H}$, Ito $\mathrm{T}$, et al. The revised Japanese clinical diagnostic criteria for chronic pancreatitis. Journal of Gastroenterology 2010;45(6):584-91. 
Sills 2006

Sills GJ. The mechanisms of action of gabapentin and pregabalin. Current Opinion in Pharmacology 2006;6(1): $108-13$.

Spanier 2013

Spanier B, Bruno MJ, Dijkgraaf MG. Incidence and mortality of acute and chronic pancreatitis in the Netherlands: a nationwide record-linked cohort study for the years 1995-2005. World Journal of Gastroenterology 2013;19(20):3018-26.
Ware 2014

Ware JE. SF-36® Health Survey Update. http://www.sf36.org/tools/sf36.shtml 2014 (accessed 8 October 2014).

Yadav 2011

Yadav D, Timmons L, Benson JT, Dierkhising RA,

Chari ST. Incidence, prevalence, and survival of chronic pancreatitis: a population-based study. American Journal of Gastroenterology 2011;106(12):2192-9.

* Indicates the major publication for the study 


\section{CHARACTERISTICS OF STUDIES}

\section{Characteristics of included studies [ordered by study ID]}

Olesen 2011

\begin{tabular}{ll}
\hline Methods & Randomised controlled trial \\
\hline Participants & Country: The Netherlands and Denmark \\
& Number randomised: 64 \\
& Post-randomisation drop-outs: 0 (0\%) \\
& Revised sample size: 64 \\
& Average age: 53 years \\
& Females: 24 (37.5\%) \\
& Length of follow-up (weeks): 3 \\
& Number of study centres: 2 \\
& Inclusion criteria \\
& 1. Diagnosis of chronic pancreatitis based on the Mayo Clinic diagnostic criteria \\
2. Chronic abdominal pain typical for pancreatitis (i.e. dull epigastric pain more than 3 \\
days per week for at least 3 months) \\
Exclusion criteria \\
1. Generalized painful conditions other than chronic pancreatitis \\
2. Pregnancy or lactation \\
3. Active (or history of) major depression \\
4. Moderate to severe renal impairment \\
5. An abnormal electrocardiogram at screening \\
6. Hypersensitivity to pregabalin or any of its components \\
\hline
\end{tabular}

Interventions

Participants were randomly assigned to two groups.

Group 1: pregabalin $(\mathrm{N}=34)$.

Further details: Initial dose: $75 \mathrm{mg}$ pregabalin twice daily, increased to $150 \mathrm{mg}$ pregabalin twice daily after 3 days, with a further increase to $300 \mathrm{mg}$ twice daily after 1 week for the rest of the study period (3 weeks).

Group 2: placebo $(\mathrm{N}=30)$.

Further details: Matching placebo

Cointervention: Patients taking concomitant analgesic medication and expected to stay on a stable regime during the trial were allowed to enter the study

Outcomes

The outcomes reported were health quality of life, opiate analgesic requirement, adverse events, pain scores, and hospital readmissions

Notes

Source of funding: "Supported by a free grant from Pfizer Research and Development, Hertha Christensen's Foundation, and Christenson-Ceson's Family Foundation”

Risk of bias

\section{Bias}

Authors' judgement

Support for judgement

Random sequence generation (selection Low risk bias)

Quote: "Randomization blocks had a size of six and were computer-generated by a pseudo-random code"

Pregabalin for decreasing pancreatic pain in chronic pancreatitis (Review)

Copyright () 2016 The Cochrane Collaboration. Published by John Wiley \& Sons, Ltd. 
Olesen 2011 (Continued)

\begin{tabular}{|c|c|c|}
\hline Allocation concealment (selection bias) & Low risk & $\begin{array}{l}\text { Quote: "Pfizer donated pregabalin and identical capsules } \\
\text { containing placebo. Patients and those administrating } \\
\text { study medication, assessing outcomes, and analyzing data } \\
\text { were blinded to group assignment" }\end{array}$ \\
\hline $\begin{array}{l}\text { Blinding of participants and personnel } \\
\text { (performance bias) } \\
\text { All outcomes }\end{array}$ & Low risk & $\begin{array}{l}\text { Quote: "Patients and those administrating study medica- } \\
\text { tion, assessing outcomes, and analyzing data were blinded } \\
\text { to group assignment" }\end{array}$ \\
\hline $\begin{array}{l}\text { Blinding of outcome assessment (detection } \\
\text { bias) } \\
\text { All outcomes }\end{array}$ & Low risk & $\begin{array}{l}\text { Quote: "Patients and those administrating study medica- } \\
\text { tion, assessing outcomes, and analyzing data were blinded } \\
\text { to group assignment" }\end{array}$ \\
\hline $\begin{array}{l}\text { Incomplete outcome data (attrition bias) } \\
\text { All outcomes }\end{array}$ & Low risk & $\begin{array}{l}\text { Comment: There were no post-randomisation drop- } \\
\text { outs. }\end{array}$ \\
\hline Selective reporting (reporting bias) & Low risk & Comment: All important outcomes were reported. \\
\hline Other bias & Low risk & Comment: No other risks of bias were present. \\
\hline
\end{tabular}


DATA AND ANALYSES

Comparison 1. Pregabalin versus placebo

\begin{tabular}{|c|c|c|c|c|}
\hline Outcome or subgroup title & $\begin{array}{l}\text { No. of } \\
\text { studies }\end{array}$ & $\begin{array}{c}\text { No. of } \\
\text { participants }\end{array}$ & Statistical method & Effect size \\
\hline $\begin{array}{l}1 \text { Health-related quality of life } \\
\text { (short-term) }\end{array}$ & 1 & & Mean Difference (IV, Fixed, 95\% CI) & Subtotals only \\
\hline 2 Serious adverse events & 1 & & Risk Ratio (M-H, Fixed, 95\% CI) & Subtotals only \\
\hline $\begin{array}{l}3 \text { Opiate analgesic requirement in } \\
\text { mg (short-term) }\end{array}$ & 1 & & Mean Difference (IV, Fixed, 95\% CI) & Subtotals only \\
\hline 4 Adverse events & 1 & & Risk Ratio (M-H, Fixed, 95\% CI) & Subtotals only \\
\hline 5 Pain scores (short-term) & 1 & & Mean Difference (IV, Fixed, 95\% CI) & Subtotals only \\
\hline 6 Hospital admissions & 1 & & Risk Ratio (M-H, Fixed, 95\% CI) & Subtotals only \\
\hline
\end{tabular}

\section{Analysis I.I. Comparison I Pregabalin versus placebo, Outcome I Health-related quality of life (short-} term).

\footnotetext{
Review: Pregabalin for decreasing pancreatic pain in chronic pancreatitis

Comparison: I Pregabalin versus placebo

Outcome: I Health-related quality of life (short-term)
}

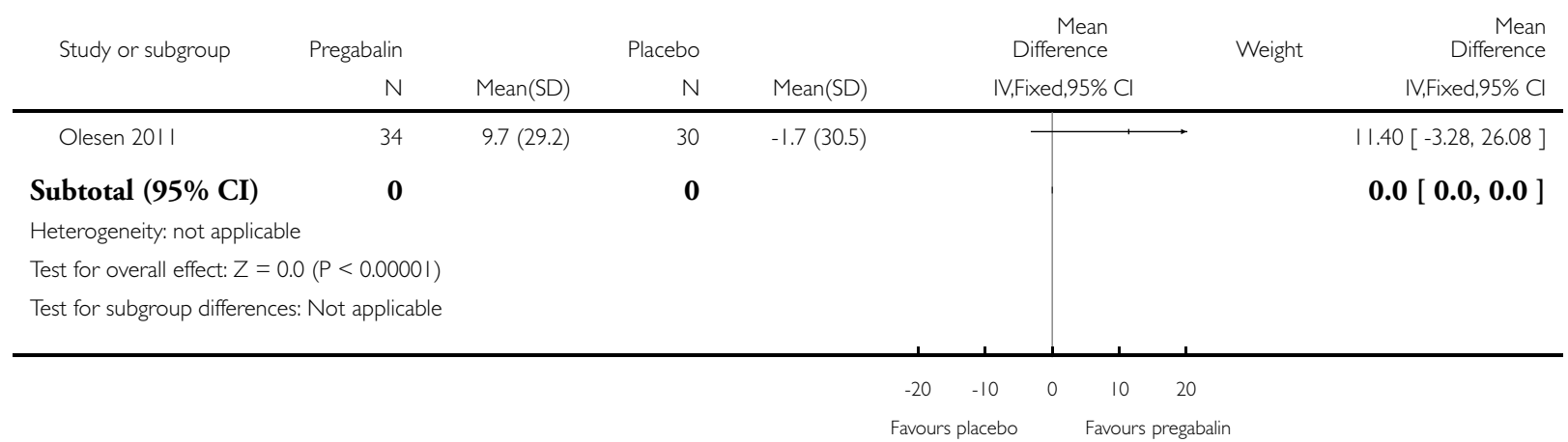




\section{Analysis I.2. Comparison I Pregabalin versus placebo, Outcome 2 Serious adverse events.}

Review: Pregabalin for decreasing pancreatic pain in chronic pancreatitis

Comparison: I Pregabalin versus placebo

Outcome: 2 Serious adverse events

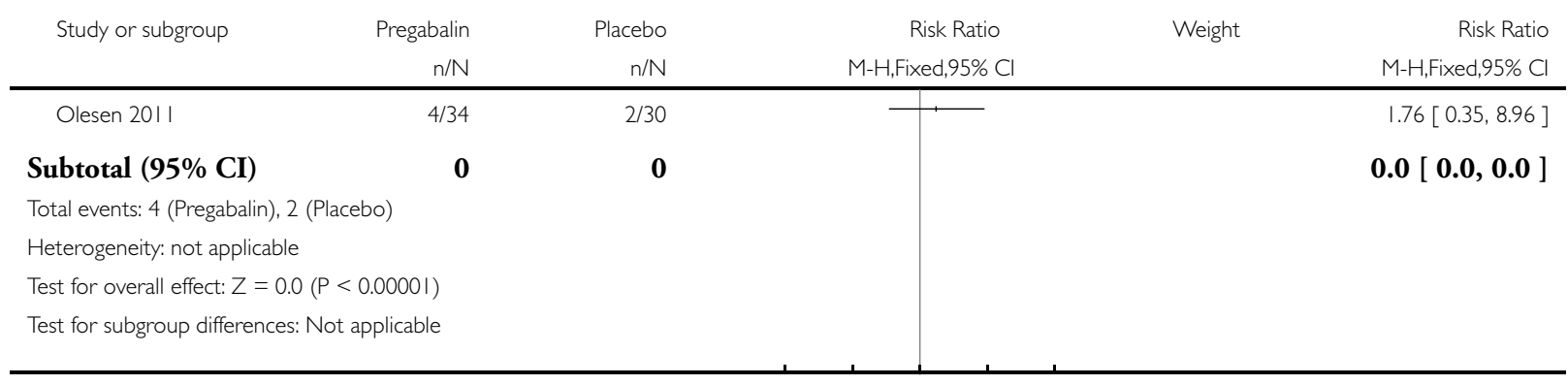

Favours pregabalin $\quad$ Favours placebo

\section{Analysis I.3. Comparison I Pregabalin versus placebo, Outcome 3 Opiate analgesic requirement in $\mathrm{mg}$} (short-term).

Review: Pregabalin for decreasing pancreatic pain in chronic pancreatitis

Comparison: I Pregabalin versus placebo

Outcome: 3 Opiate analgesic requirement in $\mathrm{mg}$ (short-term)

\begin{tabular}{|c|c|c|c|c|c|c|c|c|c|}
\hline \multirow[t]{2}{*}{ Study or subgroup } & \multirow{2}{*}{$\begin{array}{r}\text { Pregabalin } \\
N\end{array}$} & \multicolumn{2}{|c|}{ Placebo } & \multicolumn{4}{|c|}{$\begin{array}{r}\text { Mean } \\
\text { Difference }\end{array}$} & \multirow[t]{2}{*}{ Weight } & \multirow{2}{*}{$\begin{array}{r}\text { Mean } \\
\text { Difference } \\
\text { IV,Fixed,95\% Cl }\end{array}$} \\
\hline & & Mean(SD) & $\mathrm{N}$ & Mean(SD) & & & $\mathrm{ed}, 95 \% \mathrm{Cl}$ & & \\
\hline Olesen 2011 & 34 & $-30(43.5)$ & 30 & $-4(43.5)$ & & & & & $-26.00[-47.36,-4.64]$ \\
\hline Subtotal (95\% CI) & $\mathbf{0}$ & & $\mathbf{0}$ & & & & & & $0.0[0.0,0.0]$ \\
\hline \multicolumn{10}{|c|}{ Heterogeneity: not applicable } \\
\hline \multicolumn{10}{|c|}{ Test for overall effect: $Z=0.0(P<0.0000 I)$} \\
\hline \multicolumn{10}{|c|}{ Test for subgroup differences: Not applicable } \\
\hline & & & & & -100 & -50 & 50 & 100 & \\
\hline \multicolumn{10}{|c|}{ Favours pregabalin } \\
\hline
\end{tabular}




\section{Analysis I.4. Comparison I Pregabalin versus placebo, Outcome 4 Adverse events.}

Review: Pregabalin for decreasing pancreatic pain in chronic pancreatitis

Comparison: I Pregabalin versus placebo

Outcome: 4 Adverse events

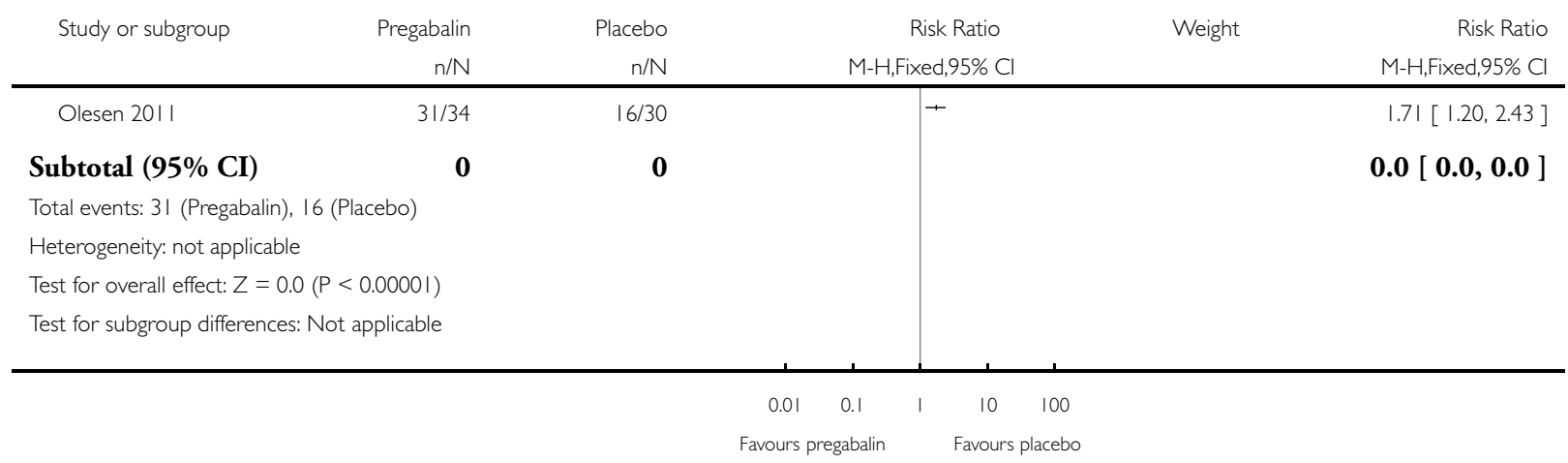

\section{Analysis I.5. Comparison I Pregabalin versus placebo, Outcome 5 Pain scores (short-term).}

Review: Pregabalin for decreasing pancreatic pain in chronic pancreatitis

Comparison: I Pregabalin versus placebo

Outcome: 5 Pain scores (short-term)






\section{Analysis I.6. Comparison I Pregabalin versus placebo, Outcome 6 Hospital admissions.}

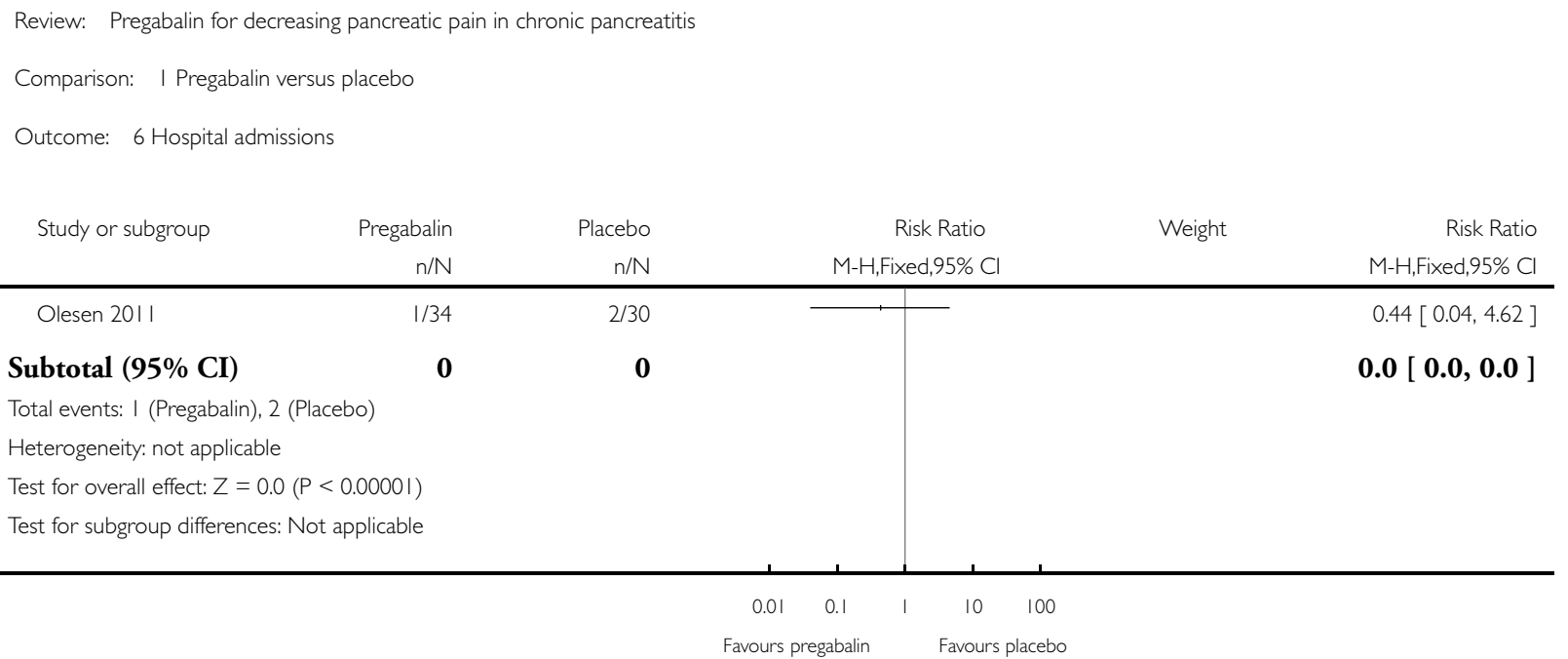

\section{A P P E N DICES}

\section{Appendix I. Glossary of terms}

Acute: sudden

Adenocarcinoma: cancer arising from glandular cells

Adjuvant: in addition to

Aetiology: cause

Analogue: a substance comparable to another substance (in this context)

Ataxia: loss of full control of body movements

Autoimmune: disease caused when the body's defence mechanism, which usually protects against infections, reacts against and damages the body's own tissues.

Biliary obstruction: blockage to flow of bile

Biopsy: examination of a piece of tissue removed from a living body

Caerulein: a hormone that has a similar action to cholecystokinin (see below)

Calcifications: calcium deposits in tissue

CFTR mutation: alteration in the genetic code for a protein called cystic fibrosis transport regulator (CFTR)

Chemotherapy: the use of medication to treat or control a disease

Cholecystokinin: a hormone that increases the contractility of the gallbladder, thus increasing the flow of bile into the small bowel

Chronic: long-standing

Coeliac plexus block: an injection of long-acting local anaesthetic into the coeliac plexus, a network of nerves that supply the abdominal organs

Computed tomography (CT scan): This is performed by taking a series of X-rays using special equipment, and processing the images using a computer to obtain a final image.

Concomitant: at the same time (in this context) 
Diabetes: a lifelong condition that causes a person's blood sugar level to become too high

Digestive enzyme deficiency: shortage of enzymes that help with digestion by breaking down the food that we eat into substances that can be absorbed from the gut

Duodenal aspirate: fluid obtained from the upper part of the small intestine, usually with a tube inserted into the small intestine through the nose or mouth

Duodenum: upper part of small bowel. It conducts digested food from the stomach to the middle part of the small bowel (jejunum). The bile duct and the pancreatic duct conduct the bile and pancreatic juice to drain into the duodenum.

Dysarthria: slurred speech

Dyspnoea: shortness of breath

Elastase: enzyme that breaks down protein

Endocrine pancreatic insufficiency: deficiency of hormones secreted from cells in the pancreas, clinically manifesting as diabetes because of insulin deficiency

Endoscopic retrograde cholangio pancreatography: a diagnostic test that involves the use of an endoscope and x-rays to image the pancreas and biliary system

Endoscopic ultrasound: an ultrasound scan that is performed using an endoscope.

Enzyme: substances (usually proteins) originating from living cells and capable of producing specific chemical changes in organic substances by catalytic action

Euphoria: feeling of high excitement

Exocrine pancreas: part of the pancreas that secretes pancreatic juice

Exocrine pancreatic insufficiency: deficiency of pancreatic digestive enzymes

Fibromyalgia: muscle or joint pain with stiffness and localised pain at specific points on the body

Gait: the way one walks

Gynaecomastia: enlargement of male breasts

High-voltage-gated calcium channels: these are activated when a nerve impulse arrives and allows movement of calcium into the cells, which in turn, activates other molecular activities, including muscle contraction and excitation of neurons.

Histopathological: examination of tissue under the microscope

Hypercalcaemia: high calcium in blood

Hyperparathyroidism: high parathormone levels (a hormone involved in maintaining calcium level) in blood

Hypertension: high blood pressure

Idiopathic: cause of disease could not be identified

Insulin: substance that helps regulate blood sugar

Libido: sexual desire

Metastatic: spread of cancer to other parts of the body

Mortality: death

Neural: nerve-related

Neurolysis: destruction of a nerve (in this context, using injection of chemicals such as $100 \%$ alcohol (absolute alcohol) or by heat generated by radiofrequency waves)

Neuropathic pain: pain due to a disease affecting the somatosensory system (part of the nerve system concerned with sensations, such as touch, pressure, pain, and temperature)

Neurotransmitter: a chemical substance released from the end of a nerve fibre when a nerve impulse arrives. Neurotransmitters are responsible for transmission of sensations and for muscle contraction.

Oedema: swelling

Pancreatic pseudocysts: fluid collections in the pancreas or the tissues surrounding the pancreas, surrounded by a well-defined wall, and containing only fluid, with little or no solid material

Parenchyma: the essential element of the organ, as opposed to the supporting tissue

Paraesthesia: tingling sensation

Pathogenesis: development of a disease

Peripancreatic: around the pancreas

Placebo: sham or dummy treatment

Prognosis: outlook (in this context, outlook for survival)

Radiotherapy: use of radiation to treat a disease

Renal: kidney

Rhabdomyolysis: destruction of voluntary muscles

Pregabalin for decreasing pancreatic pain in chronic pancreatitis (Review)

Copyright @ 2016 The Cochrane Collaboration. Published by John Wiley \& Sons, Ltd. 
Secretin enhanced magnetic resonance cholangiopancreatography: a magnetic resonance scan (MRI) performed after administering secretin (a hormone that regulates the secretion of the stomach and pancreas)

Sensory fibres: nerve fibres that carry sensations, including pain

Somnolence: sleepiness

SPINK 1 mutation: alteration in the genetic code for a protein called serine protease inhibitor Kazal-type 1 (SPINK 1)

Steatorrhoea: bulky, foul smelling stools, due to the presence of excessive fat in the stools

Surgical resection: surgical removal

Sympathetic fibres: nerve fibres that control involuntary actions in the body, such as control of blood pressure

Systemic: all over the body (in this context)

Thiazide: a medicine used to lower blood pressure by increasing the excretion of salt and water from body

Urticaria: rash of round, red wheals on the skin that itch intensely

Valproate: a medicine used in people with epilepsy

Vertigo: a sensation of spinning of the head or the environment and loss of balance

\title{
Appendix 2. CENTRAL search strategy
}

\author{
\#1 pancreas \\ \#2 pancrea* \\ \#3 MeSH descriptor: [Pancreas] explode all trees \\ \#4 chronic pancreatitis \\ \#5 MeSH descriptor: [Pancreatitis, Chronic] explode all trees \\ $\# 6$ \#1 or \#2 or \#3 or \#4 or \#5 \\ $\# 7$ pregabalin or Lyrica \\ \#8 \#6 and \#7
}

\section{Appendix 3. MEDLINE search strategy}

1. randomized controlled trial.pt.

2. controlled clinical trial.pt.

3. randomized.ab.

4. placebo.ab.

5. drug therapy.fs.

6. randomly.ab.

7. trial.ab.

8. groups.ab.

9. 1 or 2 or 3 or 4 or 5 or 6 or 7 or 8

10. exp animals/ not humans.sh.

11. 9 not 10

12. exp Pancreatitis, Chronic/

13. chronic pancreatitis.mp.

14. 12 or 13

15. exp Pancreaticoduodenectomy/

16. exp Pancreatectomy/

17. (pancreaticoduodenectomy or pancreaticoduodenectomies or duodenopancreatectomy or duodenopancreatectomies or pancreatectomy).mp.

18. 15 or 16 or 17

19. 11 and 14 and 18

Pregabalin for decreasing pancreatic pain in chronic pancreatitis (Review) 


\section{Appendix 4. EMBASE search strategy}

1. (pancreas or pancrea*).mp.

2. exp pancreas/

3. chronic pancreatitis.mp.

4. exp Pancreatitis, Chronic/

5. 1 or 2 or 3 or 4

6. (pregabalin* or Lyrica).mp.

7. exp pregabalin/

8.6 or 7

9.5 and 8

10. Clinical trial/

11. Randomized controlled trial/

12. Randomization/

13. Single-Blind Method/

14. Double-Blind Method/

15. Cross-Over Studies/

16. Random Allocation/

17. Placebo/

18. Randomi?ed controlled trial*.tw.

19. Rct.tw.

20. Random allocation.tw.

21. Randomly allocated.tw.

22. Allocated randomly.tw.

23. (allocated adj2 random).tw.

24. Single blind*.tw.

25. Double blind*.tw.

26. ((treble or triple) adj blind*).tw.

27. Placebo*.tw.

28. Prospective study/

29. or $/ 10-28$

30. Case study/

31. Case report.tw.

32. Abstract report/ or letter/

33. or $/ 30-32$

34.29 not 33

35.9 and 34

\section{Appendix 5. Science Citation Index search strategy}

\# 1 TS=(chronic pancreatitis or pancreas or pancrea*)

\# 2 TS=(pregabalin* or Lyrica)

\# 3 TS=(random* OR rct* OR crossover OR masked OR blind* OR placebo* OR meta-analysis OR systematic review* OR metaanalys*)

\# 4 \#3 AND \#2 AND \#1

Pregabalin for decreasing pancreatic pain in chronic pancreatitis (Review)

Copyright $\odot 2016$ The Cochrane Collaboration. Published by John Wiley \& Sons, Ltd. 


\section{Appendix 6. ClinicalTrials.gov search strategy}

"Interventional" [STUDY-TYPES] AND ("Phase 2" OR "Phase 3" OR "Phase 4") [PHASE] | chronic pancreatitis | pregabalin

\section{Appendix 7. WHO ICTRP search strategy}

pancrea* AND pregabalin

\section{CONTRIBUTIONSOFAUTHORS}

Conceiving the review: $\mathrm{KG}$

Designing the review: KG

Co-ordinating the review: KG

Designing search strategies: KG

Data extraction: KG, CL

Writing the review: KG

Providing general advice on the review: BRD

Securing funding for the review: KG

Performing previous work that was the foundation of the current study: KG

\section{DECLARATIONSOF INTEREST}

This report is independent research funded by the National Institute for Health Research (NIHR Cochrane Programme Grants, 13/ 89/03 - Evidence-based diagnosis and management of upper digestive, hepato-biliary, and pancreatic disorders). The views expressed in this publication are those of the author(s) and not necessarily those of the NHS, the National Institute for Health Research or the Department of Health.

KG: none known.

CL: none known.

BRD: none known.

\section{SOURCES OF SUPPORT}

\section{Internal sources}

- University College London, UK. 


\section{External sources}

- National Institute for Health Research, UK.

This project was supported by the National Institute for Health Research, via Cochrane Programme Grant to the CHBG and UGPD groups. The views and opinions expressed therein are those of the authors and do not necessarily reflect those of the Systematic Reviews Programme, NIHR, NHS or the Department of Health.

\section{DIFFERENCES BETWEEN PROTOCOLANDREVIEW}

We revised the time period of short-term outcome from 'four weeks to three months' to 'two weeks to three months' to include the immediate benefits of pregabalin.

We assessed the hospital admissions as a binary outcome, since the only trial included in this review reported the number of participants requiring readmission rather than the number of hospital admissions. 